\title{
Low Power Output-Capacitorless Class-AB CMOS LDO Regulator
}

\author{
Vahideh Shirmohammadli, Alireza Saberkari \\ Microelectronics Research Lab. \\ Department of Electrical Engineering, University of Guilan \\ Rasht, Iran \\ shirmohamadi@msc.guilan.ac.ir, a_saberkari@guilan.ac.ir
}

\author{
Herminio Martínez-García, Eduard Alarcón-Cot \\ Department of Electronics Engineering \\ Technical University of Catalunya (UPC), BarcelonaTech \\ Barcelona, Spain \\ \{herminio.martinez; eduard.alarcon\}@upc.edu
}

\begin{abstract}
This paper presents an output-capacitorless classAB low-dropout (LDO) regulator with load current sinking and sourcing ability. The proposed LDO consists of two complementary pass transistors, controlled using a level shifter technique. The transient improvement section applied to the gates of the pass devices enhances the transient performance of the LDO. The proposed LDO is designed in TSMC $0.18 \mu \mathrm{m}$ CMOS process with input and output voltages of 1.2-2.5 $\mathrm{V}$ and 1 $\mathrm{V}$, respectively, $10 \mathrm{pF}$ output capacitor, and quiescent current of $3.14 \mu \mathrm{A}$, and is capable to sink and source maximum load currents of $\pm 100 \mathrm{~mA}$, giving the current efficiency of $99.99 \%$.
\end{abstract}

Index Terms-low-dropout regulator, class-AB LDO, complementary pass transistors, level shifter, sink and source.

\section{INTRODUCTION}

$\mathrm{L}$ INEAR-ASSISTED/LDO-Assisted buck converters become an efficient solution in high speed power management circuits [1-3]. They can provide higher efficiencies rather than linear/LDO regulators and higher speed performance rather than buck converters. One of the main requirements for designing such a structure is utilization of class-AB linear/LDO regulators. Class-AB linear regulators, which are widely used and well known, usually have a push-pull amplifier as their last stage. In contrast, class-AB LDOs have been presented in limited literatures $[4,5]$. Since the design of class-AB LDO is a little bit different from its class-A counterpart, due to utilizing two complementary pass transistors (CPTs) in the common-source configuration (ntype and p-type), the design procedure implies the use of extra circuitry to control the PTs. In [4], a class-AB regulator with minimum dropout voltage of $350 \mathrm{mV}$ and large off-chip output capacitor of $30 \mu \mathrm{F}$ was proposed, which applies two current mirrors to control the operation of the CPTs. Since the upper PT, sourcing the current to the load, has a source follower structure, resulting in a relatively high difference between input and output voltages, it cannot be categorized in the class of LDO regulators, where their power transistors are in common-source configuration. In [5], the design procedure of an external capacitorless class-AB LDO with push-pull common-source power transistors was presented, in which cross-over inverters were utilized to separate and control the gate of CPTs. In addition, a quiescent current reduction circuitry was embedded into the LDO to reduce the quiescent current to a low level of $1.8 \mu \mathrm{A}$. However, it suffers from utilizing relatively large on-chip capacitances to improve the performance of the LDO.

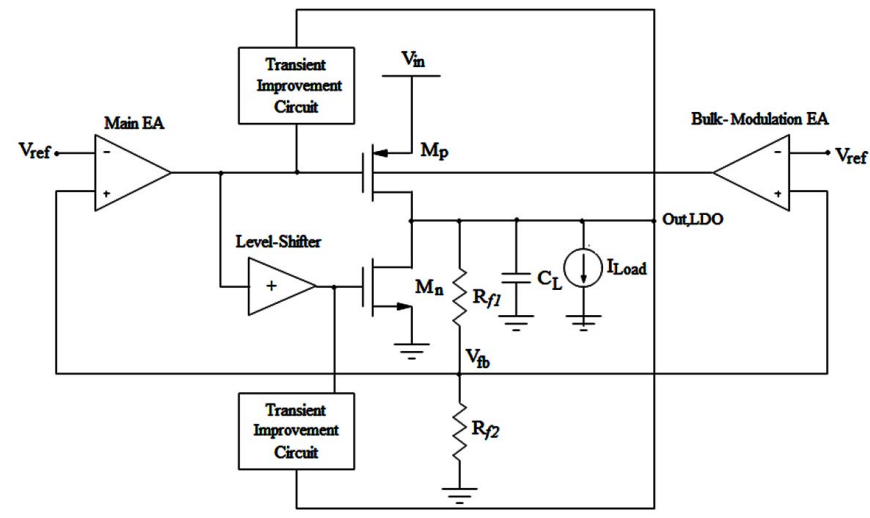

Fig. 1. Block diagram of the proposed class-AB LDO regulator.

In this paper, a new structure is proposed to control the CPTs of a fully on-chip class-AB LDO regulator based on level shifting the gate of n-type PT. Utilizing the transient improvement circuits results in enhancing the transient performance of the LDO. In addition, bulk modulation technique is applied for the p-type PT to further improve the transient behavior during the load step up and down at positive load currents [6]. The rest of the paper is as follows; in section II, the proposed LDO and its performance from the stability and transient points of view are presented. Section III belongs to the results and discussion. Finally, the achievements of the manuscript are concluded in section IV.

\section{PROPOSED Class-AB LDO REGULATORS}

Block diagram of the proposed class-AB LDO regulator is illustrated in Fig. 1. A level shifter stage is used to separate the gate of CPTs. Two transient improvement circuits are exploited to improve the transient response; in addition, the bulk modulation technique is applied for further improving the transient response at load step up and down at positive range.

The circuit level realization of the proposed LDO is shown in Fig. 2. It consists of a recycling folded cascode amplifier, $M_{1}-M_{7}$, as the main EA, which directly drives the PMOS PT, $M_{p}$, simple differential amplifier, $M_{16}$ and $M_{17}$, as the bulk modulation EA, and a level shifter, $M_{8}$ and $M_{9}$, for driving the NMOS PT, $M_{n}$. Transistors $M_{18}-M_{29}$ along with the transient coupling capacitors $C_{1}$ and $C_{2}$ form the two transient improvement circuits. The rest of the transistors are for bias. $C_{L}$ and $C_{C}$ are the on-chip load and compensation capacitors, respectively. $R_{Z}, R_{f 1}$, and $R_{f 2}$ are the compensation and feedback resistors, respectively. 


\section{A. CPT Control Technique: The Level Shifter}

A single-ended differential amplifier can be assumed as an amplifier with two inverse outputs. Each output can be used to control the gate of CPTs; the high impedance output is connected to the gate of the p-type PT and the low impedance one to the gate of the n-type PT. However, the low impedance output would not create enough voltage to pull up and down the gate of the n-type pass transistor. Hence, an inverting single-ended amplifier is utilized between the low impedance output node and the gate of the n-type PT. Thus, whole the low impedance output and the inverting amplifier are considered as the level shifter, which indeed shifts the voltage level at the gate of the n-type PT. In this case, the dimensions of the level shifter transistors, $M_{8}$ and $M_{9}$, determine the different gate voltage levels.

\section{B. Stability Analysis}

The small signal block diagram of the proposed class-AB LDO regulator is shown in Fig. 3. In general, there are four left half plane (LHP) poles, three LHP zeros, and two right half plane (RHP) zeros as below:

$$
\begin{gathered}
\omega_{p, 1}=\frac{1}{R_{e q 1} C_{e q 1}}, \omega_{p, 2}=\frac{1}{R_{e q 2} C_{e q 2}}, \omega_{p, 3}=\frac{1}{R_{e q 3} C_{e q 3}} \\
\omega_{p, 4}=\frac{1}{R_{\text {out }} C_{L}}, \quad \omega_{z, R H P 1}=\frac{g_{m p}}{C_{g d p}}, \quad \omega_{z, R H P 2}=\frac{g_{m n}}{C_{g d n}} \\
\omega_{z, L H P 1}=\frac{1}{\left(R_{z}-\frac{1}{g_{m n}}\right) C_{C}}, \omega_{z, L H P 2,3}=f\left(\omega_{p, 1}, \omega_{p, 2}, \omega_{p, 3}\right)
\end{gathered}
$$

where $R_{e q 1}, R_{e q 2}$, and $R_{e q 3}$ are the resistances seen from the output of the main EA, level shifter, and bulk modulation EA, respectively. $R_{\text {out }}$ is the resistance at the output of the LDO $\left(R_{\text {out }}=R_{L}\left\|\left(R_{f 1}+R_{f 2}\right)\right\| r_{d p} \| r_{d n}\right) . \quad G_{m 1}$ and $G_{m 3}$ are the transconductance of the main EA and bulk modulation EA, while $G_{m 2}$ is the transconductance of the low impedance path of the main EA multiplying by the level shifter transconductance. Transconductance and gate-drain capacitance of the $p$-type and $n$-type PTs are represented by $g_{m p}, g_{m n}, C_{g d p}$, and $C_{g d n}$, respectively, generating the RHP zeros, whereas $g_{m b}$ is bulk transconductance of the $p$-type PT. Two LHP zeros are resulted from two parallel paths, and can be described as a function of the LHP poles of corresponding paths, $\omega_{p 1}, \omega_{p 2}$, and $\omega_{p 3}$. The other LHP zero comes from the simple Miller compensation technique for the LDO stability achievement. Having four LHP poles and three LHP zeros makes it easier to set the stability of the LDO regulator for every load current.

\section{Transient Analysis}

Case I- Transients from zero to negative load currents:

When the load current changes suddenly from zero to negative current, the gate of the n-type PT cannot sense the changes immediately. Thus, the current is provided through charging the load capacitance. When the output voltage increases a little bit, the current of $M_{25}$ will be increased, pulling up the gate of $M_{27}$, allowing more current pass through it. As a consequence, the large current of $M_{29}$ will charge the gate of the n-type PT, resulting in passing more current through it. On the other hand, the main EA keeps off the p-type PT.

Case II- Transients from negative (positive) to zero load currents:

When the load current changes suddenly from negative

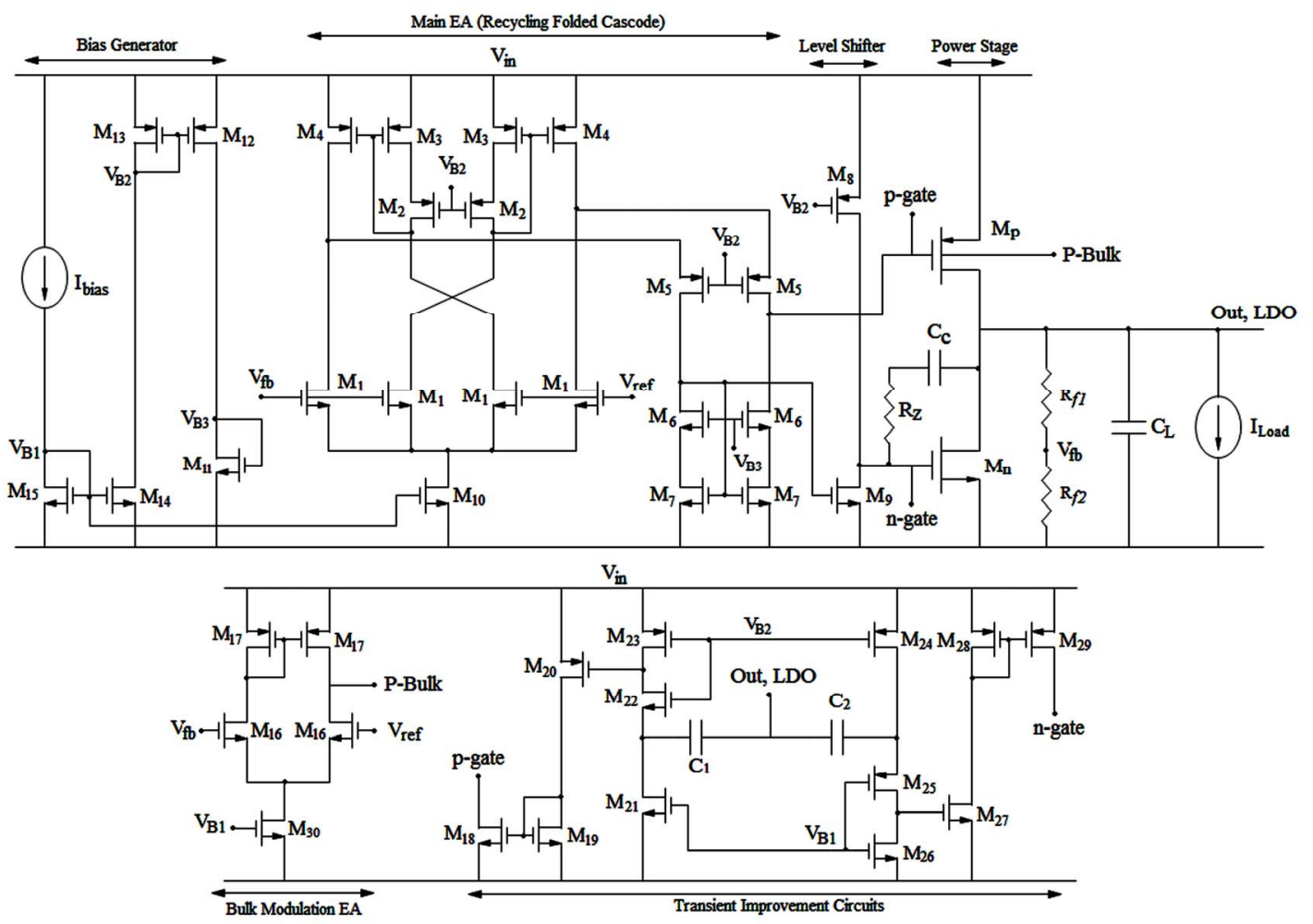

Fig. 2. Circuit level realization of the proposed class-AB LDO regulator. 


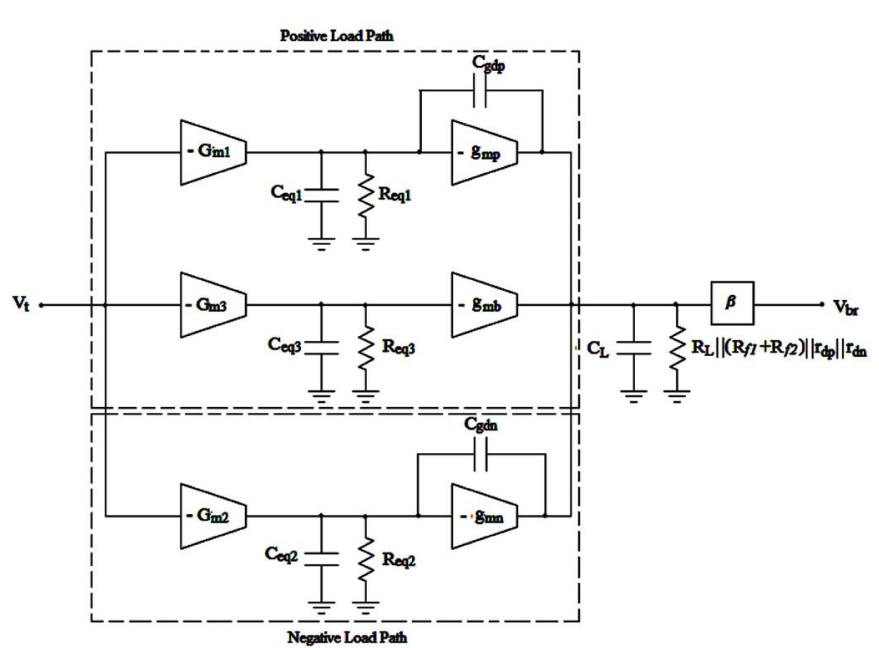

Fig. 3. Small signal block diagram of the proposed class-AB LDO regulator.

(positive) current to zero, first, the current discharges (charges) the load capacitance, decreasing (increasing) the output voltage a little bit. As a consequence, the EA will pull down (up) immediately the gate of the n-type (p-type) PT, and the process is fast enough so that there is no need for any transient improvement circuits

\section{Case III- Transients from zero to positive load currents:}

When the load current changes suddenly from zero to positive current, the gate of the p-type PT cannot sense the changes, immediately. Thus, the current is provided through discharging the load capacitance. When the output voltage decreases a little bit, the current of $M_{22}$ and $M_{20}$ will be increased, allowing more current pass through $M_{18}$. As a result, the large current of $M_{18}$ will discharge the gate of the p-type PT, resulting in pumping more current by it. In addition, the bulk modulation EA will decrease the threshold voltage of the p-type PT, increasing the current passing through it. On the other hand, the main EA keeps off the n-type PT.

\section{RESULTS AND DISCUSSION}

The proposed class-AB LDO regulator is designed and post-simulated using HSPICE in TSMC $0.18 \mu \mathrm{m}$ CMOS process. The input and output voltages are $1.2 \mathrm{~V}$ and $1 \mathrm{~V}$, respectively. The proposed LDO has a wide range of input varying from $1.2 \mathrm{~V}$ to $2.5 \mathrm{~V}$. The maximum sink and source load currents of $\pm 100 \mathrm{~mA}$ is provided by the proposed LDO, while the quiescent current is equal to $3.14 \mu \mathrm{A}$. The capacitors $C_{L}, C_{C}, C_{1}$, and $C_{2}$ are $10 \mathrm{pF}, 1 \mathrm{pF}, 0.5 \mathrm{pF}$, and $1 \mathrm{pF}$, respectively; giving the total on-chip capacitance of $12.5 \mathrm{pF}$. $R_{Z}, R_{f 1}$, and $R_{f 2}$ are $2 \mathrm{k} \Omega, 200 \mathrm{k} \Omega$, and $800 \mathrm{k} \Omega$, respectively. The layout of the proposed regulator is shown in Fig. 4 . The total core area of the chip is equal to $0.04 \mathrm{~mm}^{2}$.

Load regulations for two input voltages of $1.2 \mathrm{~V}$ and $1.8 \mathrm{~V}$ are shown in Fig. 5 (a), which are equal to $23 \mu \mathrm{V} / \mathrm{mA}$ and 15 $\mu \mathrm{V} / \mathrm{mA}$, respectively. Line regulation is provided for three different load currents of 0 and $\pm 100 \mathrm{~mA}$ in Fig. 5 (b). It has the amounts of $1.15 \mathrm{mV} / \mathrm{V}, 0.69 \mathrm{mV} / \mathrm{V}$, and $2.23 \mathrm{mV} / \mathrm{V}$ for load currents of $0,100 \mathrm{~mA}$, and $-100 \mathrm{~mA}$, respectively.

Load transient response of the LDO with load current rise and fall times of $1 \mu \mathrm{s}$ are shown in Fig. 6. The maximum

overshoot/undershoot and settling time are $220 \mathrm{mV}$ and $3.6 \mu \mathrm{s}$, respectively, while, the minimum ones are $70 \mathrm{mV}$ and $2 \mu \mathrm{s}$, respectively. In addition, line transients for the load currents of $\pm 100 \mathrm{~mA}$ and their corresponding settling times and output voltage deviations are shown in Fig. 7.

Frequency response of the proposed class-AB LDO is shown in Fig. 8 for different load currents. The phase margin varies from 35 to 85 degrees for different currents, indicating a stable treatment of the LDO for all current ranges. Furthermore, power supply rejection ratio (PSR) for different load currents is shown in Fig. 9, which varies between -50 dB

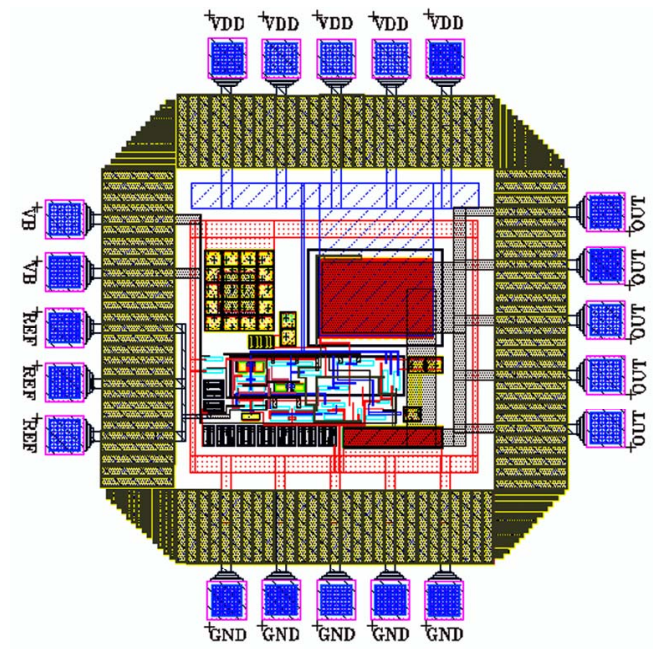

Fig. 4. Layout of the proposed class-AB LDO regulator.
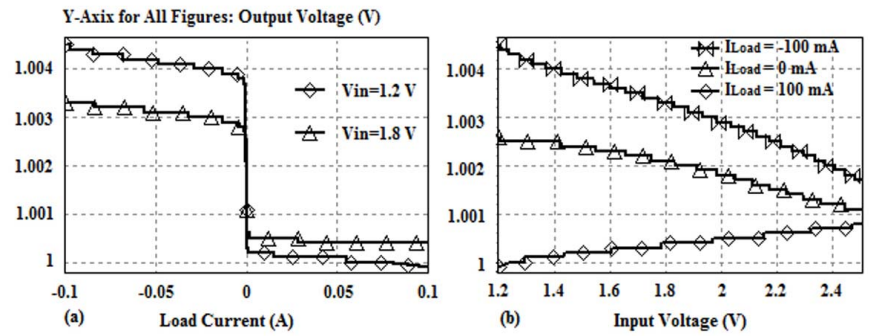

Fig. 5. (a) load regulation and (b) line regulation of the class-AB LDO.

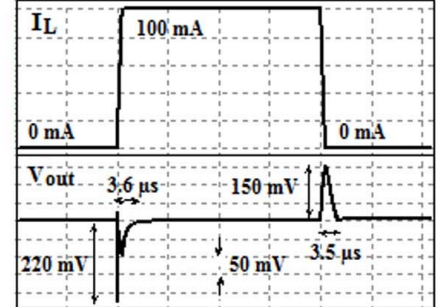

(a)

Fig. 6. Load transient behavior of the class-AB LDO for (a) positive and (b) negative loads.
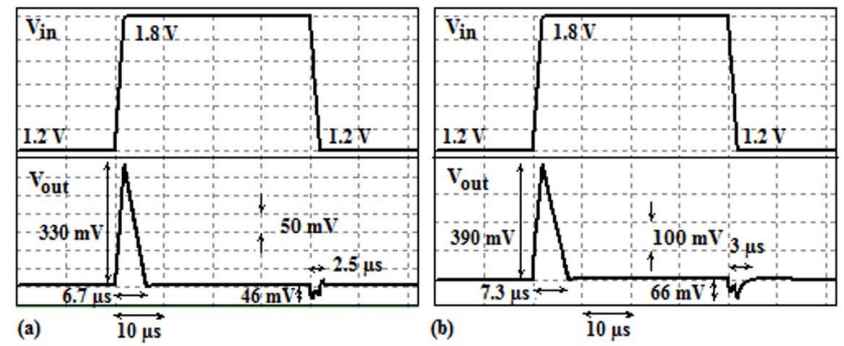

Fig. 7. Line transients for load currents of (a) $-100 \mathrm{~mA}$ and (b) $100 \mathrm{~mA}$. 


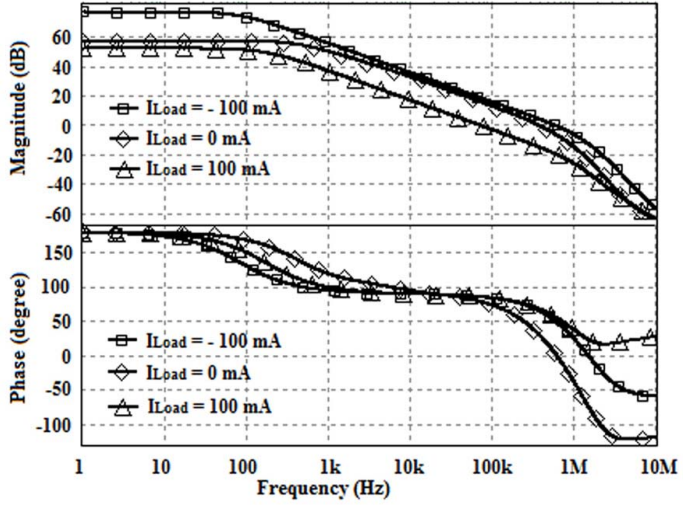

Fig. 8. Frequency response of the class-AB LDO for different load currents.

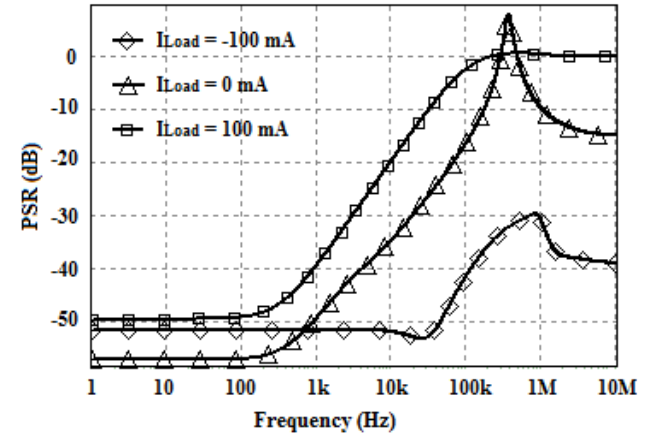

PSR@ $\mathrm{I}_{\mathrm{L}}=0 \mathrm{~mA}$ : (a) $1 \mathrm{~Hz}:-57.3 \mathrm{~dB}$ (a) $10 \mathrm{kHz}:-34.8 \mathrm{~dB}$

PSR @ I $\mathrm{L}=100 \mathrm{~mA}:$ (a) $1 \mathrm{~Hz}:-49.6 \mathrm{~dB}$ (a) $10 \mathrm{kHz}:-20 \mathrm{~dB}$

PSR@ $\mathrm{I}_{\mathrm{L}}=-100 \mathrm{~mA}$ (a) $1 \mathrm{~Hz}:-51.6 \mathrm{~dB}$ (a) $10 \mathrm{kHz}:-52 \mathrm{~dB}$

Fig. 9. PSR of the class-AB LDO in different load currents.

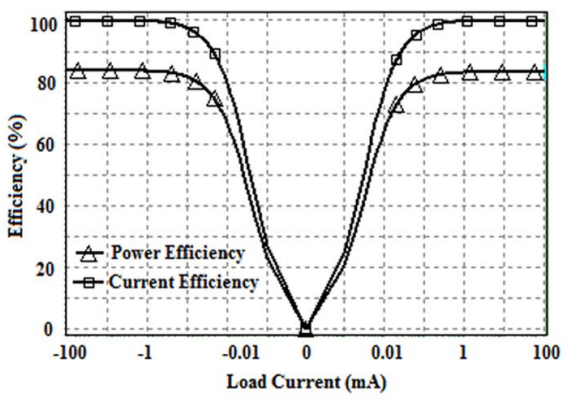

Fig. 10. Current and power efficiencies of the class-AB LDO.

to $-40 \mathrm{~dB}$ at $1 \mathrm{kHz}$ for different load currents. The current and power efficiencies of the LDO are shown in Fig. 10. The maximum current and power efficiencies of $99.99 \%$ and $83.3 \%$ are achieved, respectively.

The specifications of some recent works (class-A and class-AB) are provided in table $\mathrm{I}$, to have a benchmark performance comparison with the proposed class-AB LDO regulator. For the proposed $\mathrm{LDO}$, the results are included and the worst case for output voltage variation $\left(\Delta V_{\text {out }}\right)$ and settling time $\left(T_{\text {settle }}\right)$ is considered. Furthermore, the figure of merit ( $\left.F O M=T_{\text {settle. }} I_{Q} / I_{\text {out }, \max }\right)$ [7] is adopted here to compare the performance of different LDOs. Lower FOMs for LDOs implies better transient performances. Besides the lowest total capacitance and area consumption of the proposed class-AB LDO regulator among all reported LDOs, it has the lowest FOM rather than the others. In addition, the proposed LDO has the lowest settling time rather than the other outputcapacitorless LDOs, i.e. references [5] and [8], and its output voltage deviation is lower than that in [5]. Although the output deviation of the class-A LDO reported in [8] is lower than the proposed LDO, its settling time and quiescent current is 1.6 and 6.4 times larger than the proposed class-AB LDO.

\section{CONCLUSION}

An output-capacitorless class-AB LDO regulator with maximum load current sinking and sourcing capability of \pm 100 $\mathrm{mA}$ for a stable output voltage of $1 \mathrm{~V}$ with a $10 \mathrm{pF}$ output capacitor and $200 \mathrm{mV}$ dropout, consuming a small area and low quiescent current of $3.14 \mu \mathrm{A}$ is presented. The level shifter-based control technique was applied to control the complementary pass transistors. Additionally, transient improvement circuits were utilized to improve the transient performances of the regulator.

TABLE I. PERFORMANCE COMPARISON

\begin{tabular}{|c|c|c|c|c|}
\hline Ref. & {$[4]$} & {$[8]$} & {$[5]$} & This Work \\
\hline Data & Exp. & Exp. & Post-Sim. & Post-Sim. \\
\hline Class Type & $\mathrm{AB}$ & $\mathrm{A}$ & $\mathrm{AB}$ & AB \\
\hline Tech $(\boldsymbol{\mu m})$ & 0.5 & 0.35 & 0.18 & $\mathbf{0 . 1 8}$ \\
\hline $\boldsymbol{V}_{\text {in }}(\boldsymbol{V})$ & $2.4-6$ & 1.8 & 1.2 & $\mathbf{1 . 2}$ \\
\hline $\boldsymbol{V}_{\text {out }}(\boldsymbol{V})$ & $0.6-1.3$ & 1.6 & 1 & $\mathbf{1}$ \\
\hline $\boldsymbol{I}_{\text {out }}(\boldsymbol{m} \boldsymbol{A})$ & \pm 3000 & 100 & \pm 80 & $\pm \mathbf{1 0 0}$ \\
\hline $\boldsymbol{I}_{\boldsymbol{q}}(\boldsymbol{\mu} \boldsymbol{A})$ & 220 & 20 & 1.8 & $\mathbf{3 . 1 4}$ \\
\hline $\boldsymbol{C}_{\text {out }}(\boldsymbol{p} \boldsymbol{F})$ & $30 \times 10^{6}$ & 100 & 1 & $\mathbf{1 0}$ \\
\hline $\boldsymbol{C}_{\text {tot. }}(\boldsymbol{p} \boldsymbol{F})$ & $30 \times 10^{6}$ & 100 & 100 & $\mathbf{1 2 . 5}$ \\
\hline $\boldsymbol{T}_{\text {settle }}(\boldsymbol{\mu s})$ & 2.8 & 9 & 11 & $\mathbf{3 . 6}$ \\
\hline$\Delta \boldsymbol{V}_{\text {out }}(\boldsymbol{m} \boldsymbol{V})$ & 30 & 97 & 227 & $\mathbf{2 2 0}$ \\
\hline FOM $(\boldsymbol{n s})$ & 0.2 & 1.8 & 0.25 & $\mathbf{0 . 1 1}$ \\
\hline Area $\left(\boldsymbol{m} \boldsymbol{m} \boldsymbol{w}^{\mathbf{2}}\right)$ & 1 & 0.15 & 0.24 & $\mathbf{0 . 0 2 2}$ \\
\hline
\end{tabular}

\section{ACKNOWLEDGMENT}

This work has been partially funded by the Spanish Ministry of Science and Innovation (project DPI2013-47799C2-2-R).

\section{REFERENCES}

[1] H. Martinez and A. Saberkari, "Linear-assisted DC/DC regulator-based current source for LED drivers," Electron. Lett., vol. 52, no. 6, pp. 437439, Mar. 2016

[2] V. Shirmohammadli, A. Saberkari, H. Martinez, and E. Alarcón, "LDOassisted vs. linear-assisted DC/DC converters: a comprehensive study and comparison," in Proc. IEEE Int. Conf. Electron. Circuits Syst. (ICECS'16), Dec. 2016, pp. 105-108.

[3] V. Shirmohammadli, A. Saberkari, H. Martinez, and E. Alarcón, "Integrated power management system based on efficient LDO-assisted DC/DC buck converter," in Proc. IEEE Int. Conf. Industrial Informatics (INDIN'16), Jul. 2016, pp. 282-285.

[4] C. Xiuqin, L. Qingwei, L. Xinquan, Y. Bing, L. Yanming, and Z. Yongrui, "A 3 A sink/source current fast transient response low-dropout Gm driven linear regulator," J. Semiconduct., vol. 32, no. 6, pp. 1-5, Jun. 2011

[5] S. Asefi, A. Saberkari, H. Martinez, and E. Alarcón, "Low-quiescent current output-capacitorless class-AB CMOS low-dropout regulator," in Proc. IEEE Int. Symp. Circuits Syst. (ISCAS'16), May 2016.

[6] K. Keikhosravy and Sh. Mirabbasi, "A 0.13- $\mu$ m CMOS low-power capacitor-less LDO regulator using bulk-modulation technique," IEEE Trans. on Circuits and Syst. I, vol. 61, no. 11, pp. 3105-3114, Oct. 2014.

[7] A. Saberkari, H. Martinez, and E. Alarcon, "Fast transient response CFA-based LDO regulator," in Proc. IEEE Int. Symp. Circuits Syst. (ISCAS'12), May 2012, pp. 3150-3153.

[8] E. N. Y. Ho and P. K. T. Mok, "A capacitor-less CMOS active feedback low-dropout regulator with slew-rate enhancement for portable on-chip application," IEEE Trans. Circuits Syst. II, vol. 57, no. 2, pp. 80-84, Feb. 2010. 